\title{
On fermionic tilde conjugation rules and thermal bosonization. Hot and cold thermofields
}

\author{
S.E. Korenblit, V.V. Semenov \\ June 15, 2018
}

\begin{abstract}
A generalization of Ojima tilde conjugation rules is suggested, which reveals the coherent state properties of thermal vacuum state and is useful for the thermofield bosonization. The notion of hot and cold thermofields is introduced to distinguish different thermofield representations giving the correct normal form of thermofield solution for finite temperature Thirring model with correct renormalization and anticommutation properties.
\end{abstract}

\section{Thermodynamics of ideal 1D gases}

From the standard course [1] it may be easily shown, that equilibrium thermodynamics of the free massless bosons in the 1- dimensional box of length $L$ coincides with that of the free massless spin $1 / 2$ fermions at the same temperature $\mathrm{k}_{B} T=1 / \varsigma$ only for both zero chemical potentials, $\mu_{(B)}=\mu_{(F)}=0$, giving a simplest example of thermal bosonization [2], for pressure $P$, densities of internal energy $\mathcal{U}$ and entropy $S$ (with $h=2 \pi \hbar, c$ - speed of light):

$$
\begin{aligned}
& P_{(B),(F)}=\frac{\mathcal{U}_{(B),(F)}}{L}=\frac{\pi^{2}}{3 \varsigma^{2} h c}, \quad \frac{S_{(B),(F)}}{\mathrm{k}_{B} L}=\frac{2 \pi^{2}}{3 \varsigma h c}, \quad \text { however, } \\
& \text { for given densities: } \quad \bar{n}_{(B)}=\frac{N_{(B)}}{L}, \quad \bar{n}_{(F)}^{ \pm}=\frac{N_{(F)}^{ \pm}}{L}: \\
& \mu_{(B)}=\frac{1}{\varsigma} \ln \left(1-e^{-\bar{n}_{(B)} \varsigma h c / 2}\right), \quad \mu^{ \pm}=\frac{1}{\varsigma} \ln \left(e^{\bar{n}_{(F)}^{ \pm} \varsigma c / 2}-1\right) .
\end{aligned}
$$


This qualitative "equilibrium" picture means that both systems for the same $\varsigma, L$ have the same $P, \mathcal{U}, S$ and also another thermodynamic potentials. The condition $\mu_{(B)}=0$ for arbitrary temperature implies an infinite boson density, $\bar{n}_{(B)} \mapsto \infty$, corresponding to specific case of thermodynamic limit $N_{(B)} \rightarrow$ $\infty, L \rightarrow \infty$ for bosonic "picture". The "equilibrium" fermion pressure (11) actually is a sum of partial ones of $N_{(F)}^{+}$fermions and $N_{(F)}^{-}$antifermions, with opposite values of chemical potentials $\mu^{ \pm}= \pm \mu_{(F)}$ and with the charge density $\mathrm{Q}_{(F)} / L[11]$ :

$P_{(F)}=P_{(F)}^{+}+P_{(F)}^{-}=\frac{\pi^{2}}{3 \varsigma^{2} h c}+\frac{\mu_{(F)}^{2}}{h c}, \quad \frac{\mathrm{Q}_{(F)}}{L}=\bar{n}_{(F)}^{+}-\bar{n}_{(F)}^{-}=\frac{2 \mu_{(F)}}{h c}$.

So, for any value of $\mu_{(F)}, \mu_{(B)}$, the "equilibrium" Gibbs potentials read:

$\mathcal{G}_{(F)}=N_{(F)}^{+} \mu^{+}+N_{(F)}^{-} \mu^{-}=\mathrm{Q}_{(F)} \mu_{(F)}=\frac{2 L \mu_{(F)}^{2}}{h c}, \quad \mathcal{G}_{(B)}=N_{(B)} \mu_{(B)}$.

Thus: $\mathcal{G}_{(F)} \Longrightarrow \mathcal{G}_{(B)}=0$, only if: $\mu_{(F)} \Rightarrow 0$, with: $\bar{n}_{(F)}^{+}=\bar{n}_{(F)}^{-}$.

Nevertheless, $\mu_{(F)}=0$, for $\bar{n}_{(F)}^{0}=2 \ln 2 /(\varsigma h c)$. We want to point out that for nonzero temperature the usual infrared regularization parameter $L$ acquires a physical meaning as a macroscopic thermodynamic parameter (11) of the real or effective "box size" of the thermodynamic system under consideration. So, the corresponding dependence requires additional care.

\section{On fermionic tilde conjugation rules}

Following to Ojima [10] let us start with simplest fermionic oscillator (for one fixed mode $k^{1}$ ), which has only two normalized states $|0\rangle$ and $|1\rangle$, with energy 0 and $\omega$, annihilated/created by fermionic operators $b, b^{\dagger}: b|0\rangle=0$, $|1\rangle=b^{\dagger}|0\rangle,\left\{b, b^{\dagger}\right\}=1,\{b, b\}=0$. The thermal vacuum appears as a normalized sum of tensor products of two independent copies of these states: $|0 \widetilde{0}\rangle=|0\rangle \otimes|\widetilde{0}\rangle,|1 \widetilde{1}\rangle=|1\rangle \otimes|\widetilde{1}\rangle$, weighted with corresponding Gibbs and relative phase exponential factors [10], so that for $\left\{b, \widetilde{b}^{\#}\right\}=0,\left(\widetilde{b}^{\#}=\widetilde{b}, \widetilde{b}^{\dagger}\right)$ :

$$
\begin{aligned}
& |0(\varsigma)\rangle_{(F)}=\frac{|0 \widetilde{0}\rangle+e^{i \Phi} e^{-\varsigma \omega / 2}|1 \widetilde{1}\rangle}{\left[\langle 0 \widetilde{0} \mid 0 \widetilde{0}\rangle+e^{-\varsigma \omega}\langle 1 \widetilde{1} \mid 1 \widetilde{1}\rangle\right]^{1 / 2}} \equiv \cos \vartheta\left(1+e^{i \Phi} \tan \vartheta b^{\dagger} \widetilde{b}^{\dagger}\right)|0 \widetilde{0}\rangle, \\
& |0(\varsigma)\rangle_{(F)}=\mathcal{V}_{\vartheta(F)}^{-1}|0 \widetilde{0}\rangle, \text { where, for: } \tan ^{2} \vartheta\left(k^{1}, \varsigma\right)=e^{-\varsigma \omega}, \quad \omega=\omega\left(k^{1}\right): \\
& \mathcal{V}_{\vartheta(F)}^{-1}=\exp \left\{e^{i \Phi} \tan \vartheta G_{+}\right\} \exp \left\{-\ln \left(\cos ^{2} \vartheta\right) G_{3}\right\} \exp \left\{-e^{-i \Phi} \tan \vartheta G_{-}\right\},
\end{aligned}
$$


with: $G_{+}=b^{\dagger} \widetilde{b}^{\dagger}, \quad G_{-}=\widetilde{b} b=\left(G_{+}\right)^{\dagger}, \quad G_{3}=\frac{1}{2}\left(b^{\dagger} b-\widetilde{b} \widetilde{b}^{\dagger}\right)$, $\left[G_{+}, G_{-}\right]=2 G_{3}, \quad\left[G_{3}, G_{ \pm}\right]= \pm G_{ \pm}, \quad$ with: $\quad G_{ \pm}=G_{1} \pm i G_{2}$,

$$
\text { thus: } \mathcal{V}_{\vartheta(F)}^{-1}=\exp \left\{\vartheta\left[e^{i \Phi} G_{+}-e^{-i \Phi} G_{-}\right]\right\}=\mathcal{V}_{-\vartheta(F)}=\mathcal{V}_{\vartheta(F)}^{\dagger} \text {, }
$$

- is a standard form of operator of the coherent state for group $S U(2)$ [3]. This observation allows to identify the algebra (11) as "quasispin" algebra [4], with the cold vacuum $|0 \widetilde{0}\rangle$ as its lowest state for representation with "quasispin" $1 / 2$, and the state $|1 \widetilde{1}\rangle$ as the highest one:

$$
\begin{aligned}
& |0 \widetilde{0}\rangle \Rightarrow\left|\frac{1}{2},-\frac{1}{2}\right\rangle, \quad|1 \widetilde{1}\rangle \Rightarrow\left|\frac{1}{2}, \frac{1}{2}\right\rangle, \\
& G_{3}\left|\frac{1}{2}, \pm \frac{1}{2}\right\rangle= \pm \frac{1}{2}\left|\frac{1}{2}, \pm \frac{1}{2}\right\rangle, \quad G_{ \pm}\left|\frac{1}{2}, \pm \frac{1}{2}\right\rangle=0 .
\end{aligned}
$$

The unique arisen arbitrary relative phase $\Phi$ reflects now the fact that the quantum state is not the vector, rather the ray. Thus, the thermal vacuum (8), as a coherent state [3], is annihilated by operator $\mathcal{V}_{\vartheta(F)}^{-1} G_{-} \mathcal{V}_{\vartheta(F)}=$ $\cos ^{2} \vartheta G_{-}+e^{i \Phi} \sin 2 \vartheta G_{3}-e^{2 i \Phi} \sin ^{2} \vartheta G_{+}=\underset{\sim}{b}(\varsigma) b(\varsigma)$, as well as by operators:

$$
\begin{aligned}
& b(\varsigma)=\mathcal{V}_{\vartheta(F)}^{-1} b \mathcal{V}_{\vartheta(F)}=b \cos \vartheta-\widetilde{b}^{\dagger} e^{i \Phi} \sin \vartheta \\
& \underset{\sim}{b}(\varsigma)=\mathcal{V}_{\vartheta(F)}^{-1} \widetilde{b} \mathcal{V}_{\vartheta(F)}=\widetilde{b} \cos \vartheta+b^{\dagger} e^{i \Phi} \sin \vartheta
\end{aligned}
$$

Up to now $\widetilde{b}^{\#}$ is only notation that does not define any operation. To fix it as an operation: $\underset{\sim}{b}(\varsigma) \mapsto \widetilde{b}(\varsigma)$, one should choose the value of $\Phi$. The popular choice $\Phi=0$ leads to complicated tilde conjugation rules for the fermionic case, different from the bosonic one [9]. The Ojima choice $\Phi=-\pi / 2$ gives the same rules for both bosonic and fermionic cases [10]. We see now that the choice $\Phi=\pi / 2$ is also good and, as well as the original Ojima's one, satisfies the properties of antilinear homomorphism and the condition $\widetilde{\widetilde{b}}(\varsigma)=$ $b(\varsigma)$. It seems very convenient for the purposes of bosonization that the tilde operation has the same properties for both Fermi and Bose cases. As a byproduct, we observe a useful interpretation of the thermal vacuum, defined by Bogoliubov transformation (8), as a coherent state, obtained by coherent $S U(2)$ rotation of vacuum states for all Fermi oscillators $\left|0_{k^{1}} \widetilde{0}_{k^{1}}\right\rangle$ as a lowest quasispin states, around one and the same unit vector $\overrightarrow{\mathrm{u}}=(\sin \Phi, \cos \Phi, 0)$ onto the different angles $=-2 \vartheta\left(k^{1}\right): \mathcal{V}_{\vartheta(F)}^{-1}=\exp [i 2 \vartheta(\overrightarrow{\mathrm{u}} \cdot \vec{G})][3]$. 
Analogous picture may be obtained for bosonic thermal Bogoliubov transformation $\mathcal{V}_{\vartheta(B)}$ leading to connection between the bosonic thermal vacuum and coherent state for the discrete series representation of group $S U(1,1)$ [3]. However, for this case the numerator in (7) contains a countable number of terms with countable number of arbitrary phases $\Phi_{n}[10]$. The coherent state of the type (9), (12) would be obtained only for countable number of coherent choices: $\Phi_{n} \mapsto n \Phi, n=0,1,2, \ldots$. We did not find a reason to prefer this choice to the usual one $\Phi_{n}=0[9,10]$.

\section{Hot and cold thermofields}

So, at finite temperature, in the framework of thermofield dynamics [9] it is necessary to double the number of degrees of freedom by providing all the fields $\Psi$ with their tilde partners $\widetilde{\Psi}$. According to [9], the resulting theory will be determined by the Hamiltonian $\widehat{H}[\Psi, \widetilde{\Psi}]=H[\Psi]-\widetilde{H}[\widetilde{\Psi}]$, where $\widetilde{H}[\widetilde{\Psi}]=H^{*}\left[\widetilde{\Psi}^{*}\right]$, with $H[\Psi]=H_{0[\Psi]}\left(x^{0}\right)+H_{I[\Psi]}\left(x^{0}\right)$, so that for Thirring model [7]: $\widetilde{H}_{I[\widetilde{\Psi}]}=H_{I[\widetilde{\Psi}]}$, and $\widetilde{H}_{0[\widetilde{\Psi}]}=-H_{0[\widetilde{\Psi}]}$. Though the substitution like (15), for the free massless Dirac thermofields, $\chi(x) \mapsto \chi(x, \varsigma)$, also does not change [9] the form of the free operator: $\widehat{H}_{0}[\chi, \widetilde{\chi}]=H_{0}[\chi]-\widetilde{H}_{0}[\widetilde{\chi}]$, these free fields, generally speaking, are not now the physical fields of this QFT model [5, 11], and, as is well known [5, 9], each term $H[\Psi]$ in $\widehat{H}[\Psi, \widetilde{\Psi}]$ must be equivalent in a weak sense to the free Hamiltonian of massless (pseudo) scalar fields $(\phi(x)), \varphi(x)$, at least, at zero temperature, $T=0$.

For any functional $\mathcal{F}[\Psi]$ of Heisenberg fields $(\mathrm{HF})$ in the given representation of physical fields $\psi(x)$, i.e. for dynamical mapping (DM) $\Psi(x)=\Upsilon[\psi(x)]$ [9] at zero temperature, being interested in the matrix elements on the thermal vacuum of the type:

$\langle 0(\varsigma)|\mathcal{F}[\Psi(x)]| 0(\varsigma)\rangle=\left\langle 0 \widetilde{0}\left|\mathcal{V}_{\vartheta} \mathcal{F}[\Psi(x)] \mathcal{V}_{\vartheta}^{-1}\right| 0 \widetilde{0}\right\rangle=\left\langle 0 \widetilde{0}\left|\mathcal{F}\left[\mathcal{V}_{\vartheta} \Psi(x) \mathcal{V}_{\vartheta}^{-1}\right]\right| 0 \widetilde{0}\right\rangle,(16)$ we come to formal mapping:

$\mathcal{V}_{\vartheta} \Psi(x) \mathcal{V}_{\vartheta}^{-1}=\Psi(x,[-] \varsigma)=\Upsilon\left[\mathcal{V}_{\vartheta} \psi(x) \mathcal{V}_{\vartheta}^{-1}\right]=\Upsilon[\psi(x,[-] \varsigma)]$,

onto the "cold" physical thermofield: $\psi(x,[-] \varsigma)=\mathcal{V}_{\vartheta} \psi(x) \mathcal{V}_{\vartheta}^{-1}$,

essentially with the same coefficient functions, as for the initial DM $\Psi(x)=$ $\Upsilon[\psi(x)]$, that, contrary to [9, 10], thus transferring so all the temperature dependence from the state (8) onto these "cold" physical thermofields. However, to compute the matrix element (16) it is necessary to substitute into 
the r.h.s. of (16), (17) the cold physical thermofields (18) again in terms of the initial physical fields $\psi(x)$ via obtained from (18) their linear combinations, analogous (but not the same!) to Eqs. (15), and reorder again the so obtained operator with respect to the initial physical fields $\psi(x)$. The same operations also convert the formal mapping (17) into temperature dependent DM over the cold vacuum $|000\rangle$, and precisely in such sense we call further the r.h.s. of (17) again as a new DM $\widehat{\Upsilon}: \Psi(x,[-] \varsigma)=\Upsilon[\psi(x,[-] \varsigma)]=$ $\Upsilon\left[\mathcal{V}_{\vartheta} \psi(x) \mathcal{V}_{\vartheta}^{-1}\right] \Rightarrow \widehat{\Upsilon}[[-] \varsigma ; \psi(x)]=\widehat{\Upsilon}\left[[-] \varsigma ; c\left(k^{1}\right), \widetilde{c}\left(k^{1}\right)\right]$, or e.g. $c\left(k^{1}\right) \mapsto b_{k^{1}}$

On the contrary, the standard computation way [9, 10] implies the substitution of the inverse to (15) linear expressions of physical fields $\psi(x)=$ $\mathcal{V}_{\vartheta} \psi(x,[+] \varsigma) \mathcal{V}_{\vartheta}^{-1}$ in terms of the "hot" physical thermofields, $\psi(x,[+] \varsigma)=$ $\mathcal{V}_{\vartheta}^{-1} \psi(x) \mathcal{V}_{\vartheta}$, given by (15), into the l.h.s. of (16) and reordering the so obtained operator with respect to this hot physical thermofield over the thermal ("hot") vacuum (8). Of course, such operations give the new DM $\widehat{\Upsilon}$ for the initial HF over this hot - thermal vacuum [9]: $\Psi(x)=\Upsilon[\psi(x)]=$ $\Upsilon\left[\mathcal{V}_{\vartheta} \psi(x,[+] \varsigma) \mathcal{V}_{\vartheta}^{-1}\right] \Rightarrow \widehat{\Upsilon}[[+] \varsigma ; \psi(x,[+] \varsigma)]=\widehat{\Upsilon}\left[[+] \varsigma ; c\left(k^{1},[+] \varsigma\right), \widetilde{c}\left(k^{1},[+] \varsigma\right)\right]$. We want to point out that this field does not equal to $\Psi(x,[+] \varsigma)=\mathcal{V}_{\vartheta}^{-1} \Psi(x) \mathcal{V}_{\vartheta}$, which will appear below as a byproduct of our further consideration. To avoid some ambiguities [12, 13] one should carefully distinguish the hot and cold physical thermofields $\psi(x,[ \pm] \varsigma)$ over corresponding vacua.

The kinematic independence of tilde-conjugate fields $\widetilde{\Psi}$ means:

$$
\left.\left\{\Psi_{\xi}(x), \widetilde{\Psi}_{\xi^{\prime}}^{\#}(y)\right\}\right|_{x^{0}=y^{0}}=0,\left.\quad\left\{\Psi_{\xi}(x), \widetilde{\Psi}_{\xi^{\prime}}^{\#}(y)\right\}\right|_{(x-y)^{2}<0}=0
$$

and corresponds to above independence of their Hamiltonians and their HEqs. This allows to consider a solution only for the one of them. Since the thermal transformations $\mathcal{V}_{\vartheta(F)}, \mathcal{V}_{\vartheta(B)}$ are not depend on coordinates and time, they can be applied directly to Eqs. (19) and zero temperature HEq of Thirring model [8], resulting 1 again to the same Eqs. (19]) and HEqs for the new $\operatorname{HF} \Psi(x,[ \pm] \varsigma)$ like (17):

$$
\begin{aligned}
& i \partial_{0} \Psi(x, \varsigma)=[\Psi(x, \varsigma), \widehat{H}[\Psi, \widetilde{\Psi}]]=\left[E\left(P^{1}\right)+g \gamma^{0} \gamma_{\nu} J_{(\Psi)}^{\nu}(x, \varsigma)\right] \Psi(x, \varsigma), \\
& 2 \partial_{\xi} \Psi_{\xi}(x, \varsigma)=-i g J_{(\Psi)}^{-\xi}(x, \varsigma) \Psi_{\xi}(x, \varsigma), \quad 2 \partial_{\xi} \widetilde{\Psi}_{\xi}(x, \varsigma)=i g \widetilde{J}_{(\widetilde{\Psi})}^{-\xi}(x, \varsigma) \widetilde{\Psi}_{\xi}(x, \varsigma),
\end{aligned}
$$

\footnotetext{
${ }^{1}$ Here: $x^{\mu}=\left(x^{0}, x^{1}\right) ; x^{0}=t ; \hbar=c=1 ; \partial_{\mu}=\left(\partial_{0}, \partial_{1}\right)$; for $g^{\mu \nu}: g^{00}=-g^{11}=1$; for $\epsilon^{\mu \nu}$ : $\epsilon^{01}=-\epsilon^{10}=1 ; \bar{\Psi}(x)=\Psi^{\dagger}(x) \gamma^{0} ; \gamma^{0}=\sigma_{1}, \gamma^{1}=-i \sigma_{2}, \gamma^{5}=\gamma^{0} \gamma^{1}=\sigma_{3}, \gamma^{\mu} \gamma^{5}=-\epsilon^{\mu \nu} \gamma_{\nu}$, where $\sigma_{i}$-Pauli matrices, and $I$ - unit matrix; $x^{\xi}=x^{0}+\xi x^{1}, 2 \partial_{\xi}=2 \partial / \partial x^{\xi}=\partial_{0}+\xi \partial_{1}$, $P^{1}=-i \partial_{1}, E\left(P^{1}\right)=\gamma^{5} P^{1}$; summation over repeated $\xi= \pm$, is nowhere implied. The label $[ \pm]$ is omitted, where it is not important: $J_{(\Psi)}^{\nu}(x, \varsigma) \mapsto \bar{\Psi}(x, \varsigma) \gamma^{\nu} \Psi(x, \varsigma)$.
} 
- for each $\xi$ - component of the field, that are also formally related to the corresponding current components as:

$$
J_{(\Psi)}^{\xi}(x, \varsigma)=J_{(\Psi)}^{0}(x, \varsigma)+\xi J_{(\Psi)}^{1}(x ; \varsigma) \longmapsto 2 \Psi_{\xi}^{\dagger}(x, \varsigma) \Psi_{\xi}(x, \varsigma) .
$$

Thus, to integrate these HEqs we can sequentially repeat all the steps of our previous works [8] for $T=0$. Applying the same arguments based on the currents conservation: $\partial_{\xi} J_{(\Psi)}^{\xi}(x, \varsigma)=0, \xi= \pm$, we come to the same weak linearization conditions (here "w" means weak equality):

$$
\begin{aligned}
& \gamma^{0} \gamma_{\nu} J_{(\Psi)}^{\nu}(x, \varsigma) \stackrel{\mathrm{w}}{\longmapsto} \frac{\beta}{2 \sqrt{\pi}} \gamma^{0} \gamma_{\nu} \widehat{J}_{(\chi)}^{\nu}(x, \varsigma), \\
& \widehat{J}_{(\chi)}^{\nu}(x, \varsigma)=\lim _{\varepsilon, \widetilde{\varepsilon}) \rightarrow 0} \widehat{J}_{(\chi)}^{\nu}(x ; \varepsilon(\widetilde{\varepsilon}), \varsigma) \equiv: J_{(\chi)}^{\nu}(x, \varsigma): \text {, with } Z_{(\chi)}(a)=1,
\end{aligned}
$$

that for the same subsequently normal ordered (renormalized) current:

$J_{(\Psi)}^{0}(x, \varsigma) \longmapsto \lim _{\widetilde{\varepsilon} \rightarrow 0} \widehat{J}_{(\Psi)}^{0}(x ; \widetilde{\varepsilon}, \varsigma)=\widehat{J}_{(\Psi)}^{0}(x, \varsigma)$,
$J_{(\Psi)}^{1}(x, \varsigma) \longmapsto \lim _{\varepsilon \rightarrow 0} \widehat{J}_{(\Psi)}^{1}(x ; \varepsilon, \varsigma)=\widehat{J}_{(\Psi)}^{1}(x, \varsigma)$,

where at first: $\widetilde{\varepsilon}^{0}=\varepsilon^{1} \rightarrow 0$, when: $\widetilde{\varepsilon}^{1}=\varepsilon^{0}, \quad \varepsilon^{2}=-\widetilde{\varepsilon}^{2}>0$, for:

$\widehat{J}_{(\Psi)}^{\nu}(x ; a, \varsigma)=Z_{(\Psi)}^{-1}(a)\left[\bar{\Psi}(x+a, \varsigma) \gamma^{\nu} \Psi(x, \varsigma)-\left\langle 0 \widetilde{0}\left|\bar{\Psi}(x+a, \varsigma) \gamma^{\nu} \Psi(x, \varsigma)\right| 0 \widetilde{0}\right\rangle\right](28$

with (the same) appropriate renormalization constant $Z_{(\Psi)}(a)$, leads again to the linearization of both equations (20), (21) in the representation of the free physical fields $\chi(x, \varsigma)$. So, that again the strong operator bosonization rules for the free field only are necessary:

$$
\begin{aligned}
& \widehat{J}_{(\chi)}^{\mu}(x, \varsigma)=\frac{1}{\sqrt{\pi}} \partial^{\mu} \varphi(x, \varsigma)=-\frac{1}{\sqrt{\pi}} \epsilon^{\mu \nu} \partial_{\nu} \phi(x, \varsigma), \\
& \widehat{J}_{(\chi)}^{-\xi}(x, \varsigma)=\frac{2}{\sqrt{\pi}} \partial_{\xi} \varphi^{\xi}\left(x^{\xi}, \varsigma\right) .
\end{aligned}
$$

The thermofields $\varphi(x, \varsigma)$ and $\phi(x, \varsigma)$ are defined in (40) below as unitarily inequivalent representations of the massless scalar and pseudoscalar KleinGordon fields: $\partial_{\mu} \partial^{\mu} \varphi(x, \varsigma)=0$, and $\partial_{\mu} \partial^{\mu} \phi(x, \varsigma)=0$, and are taken mutually dual and coupled by the symmetric integral relations: $\varepsilon(s)=\operatorname{sgn}(s)$,

$$
\left.\begin{array}{l}
\phi(x, \varsigma) \\
\varphi(x, \varsigma)
\end{array}\right\}=-\frac{1}{2} \int_{-\infty}^{\infty} d y^{1} \varepsilon\left(x^{1}-y^{1}\right) \partial_{0}\left\{\begin{array}{l}
\varphi\left(y^{1}, x^{0}, \varsigma\right) \\
\phi\left(y^{1}, x^{0}, \varsigma\right)
\end{array}\right.
$$


that implies the conditions: $\varphi\left(-\infty, x^{0}, \varsigma\right)+\varphi\left(\infty, x^{0}, \varsigma\right)=\phi\left(-\infty, x^{0}, \varsigma\right)+$ $\phi\left(\infty, x^{0}, \varsigma\right)=0$. The corresponding conserved charges read:

$$
\begin{aligned}
& \left.\begin{array}{c}
O(\varsigma) \\
O_{5}(\varsigma)
\end{array}\right\}=\lim _{L \rightarrow \infty} \int_{-\infty}^{\infty} d y^{1} \Delta\left(\frac{y^{1}}{L}\right) \partial_{0}\left\{\begin{array}{c}
\varphi\left(y^{1}, x^{0}, \varsigma\right) \\
\phi\left(y^{1}, x^{0}, \varsigma\right)
\end{array}\right\} \\
& \Longrightarrow\left\{\begin{array}{l}
\phi\left(-\infty, x^{0}, \varsigma\right)-\phi\left(\infty, x^{0}, \varsigma\right) \\
\varphi\left(-\infty, x^{0}, \varsigma\right)-\varphi\left(\infty, x^{0}, \varsigma\right),
\end{array}\right.
\end{aligned}
$$

where $\Delta\left(y^{1} / L\right)$ is the volume cut-off regularization function with the Fourier image $\delta_{L}\left(k^{1}\right)(\underline{62})$. Right $(\xi=-)$ and left $(\xi=+)$ thermofields $\varphi^{\xi}\left(x^{\xi}, \varsigma\right)$ and their charges $Q^{\xi}(\varsigma)$ are defined by similar to [5] linear combinations:

$$
\begin{aligned}
& \varphi^{\xi}\left(x^{\xi}, \varsigma\right)=\frac{1}{2}[\varphi(x, \varsigma)-\xi \phi(x, \varsigma)], \text { for: } \xi= \pm, \\
& Q^{\xi}(\varsigma)=\frac{1}{2}\left[O(\varsigma)-\xi O_{5}(\varsigma)\right]= \pm 2 \varphi^{\xi}\left(x^{0} \pm \infty, \varsigma\right),
\end{aligned}
$$

These fields obey the temperature independent commutation relations:

$$
\begin{aligned}
& {\left.\left[\varphi(x, \varsigma), \partial_{0} \varphi(y, \varsigma)\right]\right|_{x^{0}=y^{0}}=\left.\left[\phi(x, \varsigma), \partial_{0} \phi(y, \varsigma)\right]\right|_{x^{0}=y^{0}}=i \delta\left(x^{1}-y^{1}\right),} \\
& {[\varphi(x, \varsigma), \varphi(y, \varsigma)]=[\phi(x, \varsigma), \phi(y, \varsigma)]=-i \frac{\varepsilon\left(x^{0}-y^{0}\right)}{2} \theta\left((x-y)^{2}\right),} \\
& {\left[\varphi^{\xi}(s, \varsigma), \varphi^{\xi^{\prime}}(\tau, \varsigma)\right]=-\frac{i}{4} \varepsilon(s-\tau) \delta_{\xi, \xi^{\prime}}, \quad\left[\varphi^{\xi}(s, \varsigma), Q^{\xi^{\prime}}(\varsigma)\right]=\frac{i}{2} \delta_{\xi, \xi^{\prime}} .}
\end{aligned}
$$

The similar commutation relations take place for their tilde-partner, that remain kinematically independent also at finite temperature: $[A(\varsigma), \widetilde{B}(\varsigma)]=$ 0 . So, up to now we cannot distinguish the hot and cold physical thermofields.

The kinematic independence of the tilde-partners fails and the difference between the hot and cold physical thermofields appears on going to the "frequency" parts of corresponding fields $\varphi^{\xi( \pm)}\left(x^{\xi}, \varsigma\right)$, and their charges $Q^{\xi( \pm)}(\varsigma)$. It manifests itself in the commutators of annihilation $(+)$ and creation $(-)$ (frequency) parts, defined by annihilation and creation operators over the initial cold vacuum $|0 \widetilde{0}\rangle$ for the pseudoscalar fields [8]: $\mathcal{P} c\left(k^{1}\right) \mathcal{P}^{-1}=-c\left(-k^{1}\right)$, $\left[c\left(k^{1}\right), c^{\dagger}\left(q^{1}\right)\right]=2 \pi 2 k^{0} \delta\left(k^{1}-q^{1}\right), c\left(k^{1}\right)|0\rangle=0, c\left(k^{1}\right)|0 \widetilde{0}\rangle=\widetilde{c}\left(k^{1}\right)|0 \widetilde{0}\rangle=0$, for both hot $[+]$, and cold $[-]$ thermofields, in the form:

$$
\begin{aligned}
& |0(\varsigma)\rangle=\mathcal{V}_{\vartheta(B)}^{-1}|0 \widetilde{0}\rangle \equiv \mathcal{V}_{(B)}[-\vartheta]|0 \widetilde{0}\rangle, \tanh ^{2} \vartheta=e^{-\varsigma k^{0}}, \vartheta=\vartheta\left(k^{1} ; \varsigma\right), \\
& \varphi(x ;[ \pm] \varsigma)=\mathcal{V}_{\vartheta(B)}^{\mp 1} \varphi(x) \mathcal{V}_{\vartheta(B)}^{ \pm 1} \Longrightarrow \varphi^{(+)}(x ;[ \pm] \varsigma)+\varphi^{(-)}(x ;[ \pm] \varsigma)
\end{aligned}
$$


and so on for all other fields $\phi(x), \varphi^{\xi}\left(x^{\xi}\right), Q^{\xi}, \ldots$, with corresponding Fourier expansions and commutators. Below we put corresponding \pm into respective brackets, and $k^{0}=\left|k^{1}\right|$ :

$$
\begin{aligned}
& \varphi^{\xi(+)}\left(x^{\xi} ;[ \pm] \varsigma\right)=-\frac{\xi}{2 \pi} \int_{-\infty}^{\infty} \frac{d k^{1}}{2 k^{0}} \theta\left(-\xi k^{1}\right)\left[\cosh \vartheta c\left(k^{1}\right) e^{-i k^{0} x^{\xi}} \mp\right. \\
& \left.\mp \sinh \vartheta \widetilde{c}\left(k^{1}\right) e^{i k^{0} x^{\xi}}\right], \quad \varphi^{\xi(-)}\left(x^{\xi} ;[ \pm] \varsigma\right)=\left\{\varphi^{\xi(+)}\left(x^{\xi} ;[ \pm] \varsigma\right)\right\}^{\dagger} \\
& \widetilde{\varphi}^{\xi(+)}\left(x^{\xi} ;[ \pm] \varsigma\right)=-\frac{\xi}{2 \pi} \int_{-\infty}^{\infty} \frac{d k^{1}}{2 k^{0}} \theta\left(-\xi k^{1}\right)\left[\cosh \vartheta \widetilde{c}\left(k^{1}\right) e^{i k^{0} x^{\xi}} \mp\right. \\
& \left.\mp \sinh \vartheta c\left(k^{1}\right) e^{-i k^{0} x^{\xi}}\right], \quad \widetilde{\varphi}^{\xi(-)}\left(x^{\xi} ;[ \pm] \varsigma\right)=\left\{\widetilde{\varphi}^{\xi(+)}\left(x^{\xi} ;[ \pm] \varsigma\right)\right\}^{\dagger} \\
& Q^{\xi(+)}([ \pm] \varsigma)=\lim _{L \rightarrow \infty} i \frac{\xi}{2} \int_{-\infty}^{\infty} d k^{1} \theta\left(-\xi k^{1}\right)\left[\cosh \vartheta c\left(k^{1}\right) e^{-i k^{0} \widehat{x}^{0}} \pm\right. \\
& \left. \pm \sinh \vartheta \widetilde{c}\left(k^{1}\right) e^{i k^{0} \widehat{x}^{0}}\right] \delta_{L}\left(k^{1}\right), \quad Q^{\xi(-)}([ \pm] \varsigma)=\left\{Q^{\xi(+)}([ \pm] \varsigma)\right\}^{\dagger} \\
& \widetilde{Q}^{\xi(+)}([ \pm] \varsigma)=\lim _{L \rightarrow \infty}-i \frac{\xi}{2} \int_{-\infty}^{\infty} d k^{1} \theta\left(-\xi k^{1}\right)\left[\cosh \vartheta \widetilde{c}\left(k^{1}\right) e^{i k^{0} \widehat{x}^{0}} \pm\right. \\
& \left. \pm \sinh \vartheta c\left(k^{1}\right) e^{-i k^{0} \widehat{x}^{0}}\right] \delta_{L}\left(k^{1}\right), \quad \widetilde{Q}^{\xi(-)}([ \pm] \varsigma)=\left\{\widetilde{Q}^{\xi(+)}([ \pm] \varsigma)\right\}^{\dagger} .
\end{aligned}
$$

Here the $\widehat{x}^{0}$ - dependence of charge frequency parts is fictitious and unphysical. It is the artifact of space regularization (32) and should be eliminated at the end of calculation. Only for hot $[+]$ thermofields one has:

$$
\begin{aligned}
& \left\langle 0(\varsigma)\left|\varphi^{\xi}(s ;[+] \varsigma) \varphi^{\xi^{\prime}}(\tau ;[+] \varsigma)\right| 0(\varsigma)\right\rangle=\left\langle 0\left|\varphi^{\xi}(s) \varphi^{\xi^{\prime}}(\tau)\right| 0\right\rangle= \\
& =\left[\varphi^{\xi(+)}(s), \varphi^{\xi^{\prime}(-)}(\tau)\right]=\frac{\delta_{\xi, \xi^{\prime}}}{i} D^{(-)}(s-\tau),
\end{aligned}
$$

(here $\left.D^{(-)}(s)=\lim _{\varsigma \rightarrow \infty} \mathcal{D}^{(-)}\left(s, \varsigma ; \mu_{1}\right)\right)$ but for both of them:

$$
\begin{aligned}
& \left\langle 0 \widetilde{0}\left|\varphi^{\xi}(s ;[ \pm] \varsigma) \varphi^{\xi^{\prime}}(\tau ;[ \pm] \varsigma)\right| 0 \widetilde{0}\right\rangle=\left[\varphi^{\xi(+)}(s ;[ \pm] \varsigma), \varphi^{\xi^{\prime}(-)}(\tau ;[ \pm] \varsigma)\right], \\
& {\left[\varphi^{\xi( \pm)}(s ;[ \pm] \varsigma), \varphi^{\xi^{\prime}(\mp)}(\tau ;[ \pm] \varsigma)\right]=( \pm 1) \frac{\delta_{\xi, \xi^{\prime}}}{i} \mathcal{D}^{(-)}\left( \pm(s-\tau), \varsigma ; \mu_{1}\right)=} \\
& =(\mp 1) \frac{1}{4 \pi} \delta_{\xi, \xi^{\prime}}\left\{\ln \left(i \bar{\mu} \frac{\varsigma}{\pi} \sinh \left(\frac{\pi}{\varsigma}( \pm(s-\tau)-i 0)\right)\right)-g\left(\varsigma, \mu_{1}\right)\right\}, \\
& {\left[\widetilde{\varphi}^{\xi( \pm)}(s ;[ \pm] \varsigma), \widetilde{\varphi}^{\xi^{\prime}(\mp)}(\tau ;[ \pm] \varsigma)\right]=(\mp 1) \frac{\delta_{\xi, \xi^{\prime}}}{i} \widetilde{\mathcal{D}}^{(-)}\left( \pm(s-\tau), \varsigma ; \mu_{1}\right)=}
\end{aligned}
$$




$$
\begin{aligned}
& =(\mp 1) \frac{1}{4 \pi} \delta_{\xi, \xi^{\prime}}\left\{\ln \left(i \bar{\mu} \frac{\varsigma}{\pi} \sinh \left(\frac{\pi}{\varsigma}(\mp(s-\tau)-i 0)\right)\right)-g\left(\varsigma, \mu_{1}\right)\right\} \\
& {\left[\varphi^{\xi( \pm)}(s ;[ \pm] \varsigma), \widetilde{\varphi}^{\xi^{\prime}(\mp)}(\tau ;[ \pm] \varsigma)\right]=} \\
& =( \pm 1)[ \pm 1] \frac{1}{4 \pi} \delta_{\xi, \xi^{\prime}}\left\{\ln \left(\cosh \left(\frac{\pi}{\varsigma}(s-\tau)\right)\right)-f\left(\varsigma, \mu_{2}\right)\right\} \\
& {\left[\varphi^{\xi( \pm)}(s ;[ \pm] \varsigma), Q^{\xi^{\prime}(\mp)}([ \pm] \varsigma)\right]=\delta_{\xi, \xi^{\prime}}\left[\frac{i}{4}-( \pm 1)\left(\frac{\widehat{x}^{0}-s}{2 \varsigma}\right)\right]} \\
& {\left[\varphi^{\xi( \pm)}(s ;[ \pm] \varsigma), \widetilde{Q}^{\xi^{\prime}(\mp)}([ \pm] \varsigma)\right]=( \pm 1)[ \pm 1] \delta_{\xi, \xi^{\prime}}\left(\frac{\widehat{x}^{0}-s}{2 \varsigma}\right)} \\
& {\left[Q^{\xi( \pm)}([ \pm] \varsigma), Q^{\xi^{\prime}(\mp)}([ \pm] \varsigma)\right]=( \pm 1) a_{1} \delta_{\xi, \xi^{\prime}}=\left[\widetilde{Q}^{\xi( \pm)}([ \pm] \varsigma), \widetilde{Q}^{\xi^{\prime}(\mp)}([ \pm] \varsigma)\right]} \\
& {\left[Q^{\xi( \pm)}([ \pm] \varsigma), \widetilde{Q}^{\xi^{\prime}(\mp)}([ \pm] \varsigma)\right]=( \pm 1)[\mp 1] a_{2} \delta_{\xi, \xi^{\prime}}=\left[\widetilde{Q}^{\xi( \pm)}([ \pm] \varsigma), Q^{\xi^{\prime}(\mp)}([ \pm] \varsigma)\right] .(58}
\end{aligned}
$$

Here the following quantities are defined: $\bar{\mu}=\mu e^{C_{\ni}}$,

$$
\begin{aligned}
& g\left(\varsigma, \mu_{1}\right)=\int_{\mu_{1}}^{\infty} \frac{d k^{1}}{k^{0}}\left(\frac{2}{e^{\varsigma k^{0}-1}}\right) \Longrightarrow \frac{2}{\varsigma \mu_{1}}-\ln \left(\frac{2 \pi}{\varsigma \bar{\mu}_{1}}\right), \quad \bar{\mu}_{1}=\mu_{1} e^{C_{\ni}} \rightarrow 0, \\
& f\left(\varsigma, \mu_{2}\right)=\int_{\mu_{2}}^{\infty} \frac{d k^{1}}{k^{0}} \frac{1}{\sinh \left(\varsigma k^{0} / 2\right)} \Longrightarrow \frac{2}{\varsigma \mu_{2}}-\ln 2, \quad \mu_{2} \rightarrow 0, \\
& \lim _{\varsigma \rightarrow \infty} g\left(\varsigma, \mu_{1}\right)=0, \quad \lim _{\varsigma \rightarrow \infty} f\left(\varsigma, \mu_{2}\right)=0, \\
& \delta_{L}\left(k^{1}\right)=\int_{-\infty}^{\infty} \frac{d x^{1}}{2 \pi} \Delta\left(\frac{x^{1}}{L}\right) e^{ \pm i k^{1} x^{1}}=L \bar{\Delta}\left(k^{1} L\right), \quad \lim _{L \rightarrow \infty} \delta_{L}\left(k^{1}\right)=\delta\left(k^{1}\right), \\
& a_{0}=\pi \int_{0}^{\infty} d k^{1} k^{1}\left(\delta_{L}\left(k^{1}\right)\right)^{2}=\pi \int_{0}^{\infty} d t t(\bar{\Delta}(t))^{2} \equiv \pi I_{1}^{\Delta}, I_{n}^{\Delta}=\int_{0}^{\infty} d t t^{n}(\bar{\Delta}(t))^{2}, \\
& a_{1}=a_{0}+2 \pi \int_{0}^{\infty} d k^{1} k^{1} \frac{\left(\delta_{L}\left(k^{1}\right)\right)^{2}}{e^{\varsigma k^{0}}-1}=a_{0}+2 \pi \int_{0}^{\infty} d t t \frac{(\bar{\Delta}(t))^{2}}{e^{\varsigma t / L}-1}, \\
& a_{1} \Longrightarrow 2 \pi I_{0}^{\Delta} \frac{L}{\varsigma}+\frac{\pi}{6} I_{2}^{\Delta} \frac{\varsigma}{L}+O\left(\left(\frac{\varsigma}{L}\right)^{3}\right), \quad L \rightarrow \infty, \quad \lim _{\varsigma \rightarrow \infty} a_{1}=a_{0}, \\
& a_{2}=\pi \int_{0}^{\infty} d k^{1} k^{1} \frac{\left(\delta_{L}\left(k^{1}\right)\right)^{2}}{\sinh \left(\varsigma k^{0} / 2\right)}=\pi \int_{0}^{\infty} d t t \frac{(\bar{\Delta}(t))^{2}}{\sinh (t \varsigma / 2 L)}, \quad \lim _{\varsigma \rightarrow \infty} a_{2}=0, \\
& a_{2} \Longrightarrow 2 \pi I_{0}^{\Delta} \frac{L}{\varsigma}-\frac{\pi}{12} I_{2}^{\Delta} \frac{\varsigma}{L}+O\left(\left(\frac{\varsigma}{L}\right)^{3}\right), \quad L \rightarrow \infty, \quad L
\end{aligned}
$$


where $C_{\ni}$ is the Euler-Mascheroni constant. It is important to note that in any case the difference $a_{1}-a_{2}$ becomes $L$ - independent at $L \rightarrow \infty$, and if $a_{0}$ is finite, then $a_{1}-a_{2} \rightarrow 0$ at $L \rightarrow \infty$.

Following [5], by the use of the fields given above, one can construct a variety of different inequivalent representations of solutions of the Dirac equation for a free massless trial field at finite temperature, $\partial_{\xi} \chi_{\xi}\left(x^{-\xi}, \varsigma\right)=0$ in the form of local normal ordered exponentials of the left and right bosonic thermofields $\varphi^{\xi}\left(x^{\xi}, \varsigma\right)$, and their charges $Q^{\xi}(\varsigma)$ (34), (35). However, the kinematic independence (19) of the tilde-partners can be achieved only by "admixing" the Klein factors of both the charges $\widetilde{Q}^{\xi}(\varsigma)$ and $\widetilde{Q}^{-\xi}(\varsigma)$ to the same field. Moreover, according to the meaning of $L$ as macroscopic parameter, the wanted thermofield should have a correct thermodynamic limit $L \rightarrow \infty$ for the finite temperature $T>0$. The most simple case, which leads to the bosonization relations (29), (30) for the currents (25) $-(28)$ of the fields $\chi(x, \varsigma)$ with $Z_{(\chi)}(a)=1$ reads at $L \rightarrow \infty$, for $a_{1}-a_{2} \rightarrow 0,(\varpi$ and $\Theta$ are arbitrary initial overall and relative phases) as:

$$
\begin{aligned}
& \chi_{\xi}\left(x^{-\xi} ;[ \pm] \varsigma\right)=\mathcal{N}_{\varphi}\left(\exp \left\{R_{\xi}\left(x^{-\xi} ;[ \pm] \varsigma\right)\right\}\right) u_{\xi}\left(\mu_{1},[ \pm] \varsigma\right) \\
& R_{\xi}\left(x^{-\xi} ;[ \pm] \varsigma\right)=-i 2 \sqrt{\pi}\left[\varphi^{-\xi}\left(x^{-\xi} ;[ \pm] \varsigma\right)+\frac{\sigma_{0}^{\xi}}{4} \mathrm{G}^{-\xi}([ \pm] \varsigma)+\frac{\sigma_{1}^{\xi}}{4} \mathrm{G}^{\xi}([ \pm] \varsigma)\right] \\
& u_{\xi}\left(\mu_{1},[ \pm] \varsigma\right)=\left(\frac{\bar{\mu}}{2 \pi}\right)^{1 / 2} e^{i \varpi-i \xi \Theta / 4} \exp \left\{-\frac{g\left(\varsigma, \mu_{1}\right)}{2}\right\} \\
& \text { with: } \sigma_{0}^{\xi}=-\xi \sigma, \quad \sigma_{1}^{\xi}=\xi 1+\rho
\end{aligned}
$$

where the $\sigma$ and $\rho$ are defined by the condition (19), and the new charges, with simple commutation relations following from (55)-(58), are used:

$$
\begin{aligned}
& \mathrm{G}^{\xi}([ \pm] \varsigma)=Q^{\xi}([ \pm] \varsigma)+[ \pm 1] \widetilde{Q}^{\xi}([ \pm] \varsigma), \text { with: } \\
& {\left[\varphi^{\xi( \pm)}(s ;[ \pm] \varsigma), \mathrm{G}^{\xi^{\prime}(\mp)}([ \pm] \varsigma)\right]=\frac{i}{4} \delta_{\xi, \xi^{\prime}},} \\
& {\left[\varphi^{\xi( \pm)}(s ;[ \pm] \varsigma), \widetilde{\mathrm{G}}^{\xi^{\prime}(\mp)}([ \pm] \varsigma)\right]=[ \pm 1] \frac{i}{4} \delta_{\xi, \xi^{\prime}},} \\
& {\left[\mathrm{G}{ }^{\xi( \pm)}([ \pm] \varsigma), \mathrm{G}^{\xi^{\prime}(\mp)}([ \pm] \varsigma)\right]=( \pm 1) 2\left(a_{1}-a_{2}\right) \delta_{\xi, \xi^{\prime}},} \\
& {\left[\mathrm{G}^{\xi( \pm)}([ \pm] \varsigma), \widetilde{\mathrm{G}}^{\xi^{\prime}(\mp)}([ \pm] \varsigma)\right]=( \pm 1)[ \pm 1] 2\left(a_{1}-a_{2}\right) \delta_{\xi, \xi^{\prime}}}
\end{aligned}
$$

Following to [8], we obtain then at $L \rightarrow \infty$ the normal exponential of the DM for Thirring field in the form analogous to [5, 6] ( $\Lambda$ is ultraviolet cut-off):

$$
\Psi_{\xi}(x ;[ \pm] \varsigma)=\mathcal{N}_{\varphi}\left(\exp \left\{\Re_{\xi}(x ;[ \pm] \varsigma)\right\}\right) w_{\xi}\left(\mu_{1}, \varsigma\right),
$$




$$
\begin{aligned}
& \Re_{\xi}(x ;[ \pm] \varsigma)=-i\left[\Xi^{-\xi}(x ;[ \pm] \varsigma)+\frac{\Sigma_{0}^{\xi}}{4} \mathrm{G}^{-\xi}([ \pm] \varsigma)+\frac{\Sigma_{1}^{\xi}}{4} \mathrm{G}^{\xi}([ \pm] \varsigma)\right] \\
& \Xi^{-\xi}(x ;[ \pm] \varsigma)=\bar{\alpha} \varphi^{-\xi}\left(x^{-\xi} ;[ \pm] \varsigma\right)+\bar{\beta} \varphi^{\xi}\left(x^{\xi} ;[ \pm] \varsigma\right), \\
& w_{\xi}\left(\mu_{1}, \varsigma\right)=\left(\frac{\bar{\mu}}{2 \pi}\right)^{1 / 2}\left(\frac{\bar{\mu}}{\Lambda}\right)^{\bar{\beta}^{2} / 4 \pi} e^{i \varpi-i \xi \Theta / 4} \exp \left\{-g\left(\varsigma, \mu_{1}\right)\left(\frac{1}{2}+\frac{\bar{\beta}^{2}}{4 \pi}\right)\right\}, \\
& \text { where: } \Sigma_{0}^{\xi}=\bar{\alpha} \sigma_{0}^{\xi}-\bar{\beta} \sigma_{1}^{\xi}, \quad \Sigma_{1}^{\xi}=\bar{\alpha} \sigma_{1}^{\xi}-\bar{\beta} \sigma_{0}^{\xi} . \\
& \text { with: } \sigma_{0}^{\xi} \Rightarrow \xi\left(2 n_{0}+1\right), \quad \sigma_{1}^{\xi} \Rightarrow \xi 1+\left(2 n_{2}+1\right), \quad n_{0}, n_{2}-\text { integer, }
\end{aligned}
$$

by imposing again the conditions onto the parameters that are necessary to have correct Lorentz-transformation properties corresponding to the spin $1 / 2$, and correct canonical anticommutation relations (86) respectively:

$$
\bar{\alpha}^{2}-\bar{\beta}^{2}=4 \pi, \quad \bar{\beta}-\frac{\beta g}{2 \pi}=0 .
$$

Remarkably, that the obtained conditions (82) provide the anticommutation (86)), locality and kinematic independence relations (19) for both the free (68) and Thirring fields (77) and their tilde partners simultaneously.

Straightforward calculation of the vector current operators (25) $-(28)$ for the solution (777)-(82), with $Z_{(\Psi)}(a)=\left(-\Lambda^{2} a^{2}\right)^{-\bar{\beta}^{2} / 4 \pi}, Z_{(\chi)}(a)=1$, by means of Eqs. (38)-(58) and (83), under the conditions [8]:

$$
\bar{\alpha}=\left(\frac{2 \pi}{\beta}+\frac{\beta}{2}\right), \quad \bar{\beta}=\left(\frac{2 \pi}{\beta}-\frac{\beta}{2}\right), \quad \text { with: } \frac{2 \sqrt{\pi}}{\beta}=\sqrt{1+\frac{g}{\pi}},
$$

again directly reproduces the bosonization (and linearization) relations (23), (24), (29), (30) as the following weak equalities:

$$
\widehat{J}_{(\Psi)}^{\nu}(x, \varsigma) \stackrel{\mathrm{w}}{=}-\frac{\beta}{2 \pi} \epsilon^{\mu \nu} \partial_{\nu} \phi(x, \varsigma)=\frac{\beta}{2 \sqrt{\pi}} \widehat{J}_{(\chi)}^{\nu}(x, \varsigma),
$$

demonstrating self-consistency of the above calculations. The obtained normal form of Thirring thermofields has a correct renormalization properties:

$$
\begin{aligned}
& \left.\left\{\Psi_{\xi}(x, \varsigma), \Psi_{\xi^{\prime}}^{\dagger}(y, \varsigma)\right\}\right|_{x^{0}=y^{0}}=\left.Z_{(\Psi)}(x-y)\right|_{x^{0}=y^{0}} \delta_{\xi, \xi^{\prime}} \delta\left(x^{1}-y^{1}\right), \\
& \text { with: }\left.Z_{(\Psi)}(x-y)\right|_{x^{0}=y^{0}}=\left[\Lambda^{2}\left(x^{1}-y^{1}\right)^{2}\right]^{-\bar{\beta}^{2} / 4 \pi} \sim 1,
\end{aligned}
$$

for $x^{1}-y^{1} \simeq 1 / \Lambda$. The limit of this solution to zero temperature generalizes Oksak solution [5, 6] as two-parametric Thirring field with arbitrary continuous parameters $\sigma$ and $\rho$ from (71). 


\section{Conclusion}

The main lesson of our work is very simple: the correct true HF should be only a fully normal ordered operator in the sense of DM onto irreducible physical fields. Only this form clarifies and assures correct renormalization, commutation and symmetry properties. It allows also a simple connections between different types of solutions with finite and zero temperature. The chosen here representation space of free massless pseudoscalar field relax the problem of nonpositivity of inner product. Contrary to the recent works [12, 13], we take into account different types of charge regularization and all possible mutual commutation relations of bosonic thermofields and their charges, that self-consistently removes fictitious $\widehat{x}^{0}$-dependence.

The authors thank Y. Frishman, A.N. Vall, S.V. Lovtsov, V.M. Leviant, and participants of seminar in LTPh JINR for useful discussions.

This work was supported in part by the RFBR (project N 09-02-00749) and by the program "Development of Scientific Potential in Higher Schools" (project N 2.2.1.1/1483, 2.1.1/1539).

\section{References}

[1] Isihara A., Statistical physics (Academic Press, New York - London, 1971).

[2] Gomez Nicóla A., Steer D., Thermal bosonization in the sine-Gordon and Massive Thirring Models. // Nucl.Phys. B549, 1999, 409-449.

[3] Perelomov A.M., Generalized coherent states and their applications (Springer, Berlin, 1986).

[4] Lipkin H.J., Quantum mechanics (North-Holland Publishing Company, Amsterdam, 1973).

[5] Bogoliubov N.N., Logunov A.A., Oksak A.I., Todorov I. T., General principles of quantum field theory (Kluwer Academic Publishers, Boston, 1990).

[6] Oksak A.I., Non-Fock linear boson systems and their applications in two-dimentional models. // Teoret. Mat. Fiz. 48, 1981, 297-318. 
[7] Dell'Antonio G.F., Frishman Y., Zwanziger D., Thirring model in terms of currents: solution and light-cone expansions. // Phys. Rev. D6, 1972, 988-1007.

[8] Korenblit S.E., Semenov V.V., Massless Thirring model in canonical quantization scheme. // J. Nonlin. Math. Phys., 18, N 1, 2011, 6574. Korenblit S.E., Semenov V.V., Integration of the Thirring model equations. // Russian Physics Journal, 53, N 6, 2010, 630-638. (arXiv: hep-th/1003.1439 v.2)

[9] Umezawa H., Matsumoto H., Tachiki M., Thermo-field dynamics and condensed states (North-Holland Publishing Company, Amsterdam, 1982).

[10] Ojima I., Gauge fields at finite temperatures: thermo field dynamics, KMS condition and their extension to gauge theories. // Annals of Physics 137, 1981, 1-32.

[11] Alvarez-Estrada R.F., Gomez Nicóla A., The Schwinger and Thirring models at finite chemical potential and temperature. // Phys. Rev. D57, 1998, 3618-3633.

[12] Amaral R.L.P.G., Belvedere L.V., Rothe K.D., Two-dimensional thermofield bosonization. // Annals of Physics, 320, 2005, 399-428.

[13] Amaral R.L.P.G., Belvedere L.V., Rothe K.D., Rodrigues A.F., Quantum electrodynamics in two dimensions at finite temperature: thermofield bosonization approach. // J.Phys. A44, 2011, 025401. 